\title{
Replacement of the ascending aorta with reduction of the diameter of the sinotubular junction to treat aortic insufficiency in patients with ascending aortic aneurysm
}

Tirone E. David, MD, Christopher M. Feindel, MD, Susan Armstrong, MSc, and Manjula Maganti, MSc

Objective: Ascending aortic aneurysm often causes dilation of the sinotubular junction with consequent aortic insufficiency despite normal aortic cusps.

Methods: Replacement of the ascending aorta with reduction of the diameter of the sinotubular junction to correct aortic insufficiency was performed in 103 consecutive patients. Twenty-nine also needed repair of cusp prolapse. The patients' mean age was $65 \pm 12$ years and all had ascending aortic aneurysm; 63 also had arch aneurysm and 21 had aneurysm of the entire aorta. The aortic insufficiency was graded as moderate in 54 patients and severe in 49 . Patients were followed up prospectively and had echocardiographic studies annually. The mean follow-up was $5.7 \pm 3.4$ years.

Results: There were 2 operative and 30 late deaths. Cardiovascular events were the cause of death in 19 patients. Arch aneurysm and age were the only predictors of late death. Overall survival at 10 years was $54 \% \pm 7 \%$. Seven patients had moderate and 1 had severe aortic insufficiency. The freedom from moderate or severe aortic insufficiency at 10 years was $80 \% \pm 7 \%$ and from severe aortic insufficiency, $98 \% \pm 1 \%$. Two patients required aortic valve replacement, 1 because of severe aortic insufficiency and 1 for endocarditis. The freedom from aortic valve replacement at 10 years was $97 \% \pm 1 \%$. At the latest follow-up, $98 \%$ of the patients were in functional class I or II.

Conclusions: Reduction in the diameter of the sinotubular junction restores aortic valve competence in patients with ascending aortic aneurysm and aortic insufficiency with normal aortic cusps. Late survival of these patients is suboptimal because they often have extensive aneurysmal disease.

From the Peter Munk Cardiac Centre at the University Health Network and University of Toronto, Toronto, Ontario, Canada.

Received for publication July 18, 2006; revisions received Sept 17, 2006; accepted for publication Sept 29, 2006.

Address for reprints: Tirone E. David, MD, 200 Elizabeth St, 4N-457, Toronto, Ontario M5G 2C4, Canada (E-mail: tirone.david@ uhn.on.ca).

J Thorac Cardiovasc Surg 2007;133:414-8

$0022-5223 / \$ 32.00$

Copyright $\odot 2007$ by The American Association for Thoracic Surgery

doi:10.1016/j.jtcvs.2006.09.049
$\mathrm{T}$ The term "aortic valve-sparing operations" was introduced in the early 1990s to describe procedures designed to preserve the aortic valve cusps in patients with aortic root aneurysm with or without aortic insufficiency (AI) or ascending aortic aneurysms with AI owing to dilation of the sinotubular junction (STJ). ${ }^{1,2}$ Aortic valve-sparing operations to treat aortic root aneurysms are complex procedures because most anatomic components of the aortic root need surgical correction. ${ }^{3}$ This is not the case in patients who have an ascending aortic aneurysm and normal or minimally dilated aortic root because the AI is usually caused by dilation of the STJ. ${ }^{4}$ Simple reduction of its diameter restores valve competence if the cusps are normal. ${ }^{4}$ This study examines the clinical and echocardiographic results of replacement of the ascending aorta with reduction of the diameter of the STJ to correct AI in patients with ascending aortic aneurysms and normal or minimally dilated aortic sinuses.

\section{Patients and Methods}

From September 1990 to June 2005, 103 consecutive patients with ascending aortic aneurysm and AI had replacement of the ascending aorta and repair of the aortic valve. Patients with mild or no AI were excluded from this study. Table 1 shows the clinical profile of the patients. 


\section{Abbreviations and Acronyms \\ $\mathrm{AI}=$ aortic insufficiency \\ STJ $=$ sinotubular junction}

\section{Operations}

The aortic valve repair consisted of simple reduction of the diameter of the STJ by suturing a tubular Dacron graft of appropriate

TABLE 1. Clinical profile of patients with dilated sinotubular junction

\begin{tabular}{|c|c|}
\hline Variable & $\begin{array}{c}\text { No. of } \\
\text { patients }(\%)\end{array}$ \\
\hline No. of patients & 103 \\
\hline Mean age $\pm S D, y$ & $65 \pm 12$ \\
\hline Male gender & $55(53)$ \\
\hline \multicolumn{2}{|l|}{ Electrocardiogram } \\
\hline Sinus rhythm & $95(92)$ \\
\hline Atrial fibrillation & $8(8)$ \\
\hline \multicolumn{2}{|l|}{ Associated diseases } \\
\hline Diabetes & $5(5)$ \\
\hline Hypertension & $65(63)$ \\
\hline High cholesterol & $22(21)$ \\
\hline Chronic obstructive lung disease & $11(11)$ \\
\hline Renal failure & $2(2)$ \\
\hline Heart failure & $33(32)$ \\
\hline Angina pectoris & $22(21)$ \\
\hline Cardiogenic shock & $6(6)$ \\
\hline \multicolumn{2}{|l|}{ New York Heart Association } \\
\hline Class I & $26(25)$ \\
\hline Class II & $32(31)$ \\
\hline Class III & $25(24)$ \\
\hline Class IV & $20(19)$ \\
\hline \multicolumn{2}{|l|}{ Aortic dissection } \\
\hline Acute & $14(13)$ \\
\hline Chronic & $3(3)$ \\
\hline Previous heart surgery & 6 \\
\hline \multicolumn{2}{|l|}{ Left ventricular ejection fraction } \\
\hline$\geq 60 \%$ & $35(34)$ \\
\hline $40 \%-59 \%$ & $51(50)$ \\
\hline $21 \%-39 \%$ & $14(13)$ \\
\hline$\leq 20 \%$ & $2(2)$ \\
\hline Unknown & $1(1)$ \\
\hline Coronary artery disease & $27(26)$ \\
\hline Mitral insufficiency & $7(7)$ \\
\hline Bicuspid aortic valve & $9(9)$ \\
\hline Aortic arch aneurysm & $63(61)$ \\
\hline Mega-aorta syndrome & $21(20)$ \\
\hline Diameter of the ascending aorta, $\mathrm{mm}$ (mean $\pm \mathrm{SD}$ ) & $65 \pm 12$ \\
\hline $\begin{array}{l}\text { Diameter of the sinotubular junction, } \mathrm{mm} \\
\quad(\text { mean } \pm \mathrm{SD})\end{array}$ & $39 \pm 9$ \\
\hline \multicolumn{2}{|l|}{ Aortic insufficiency } \\
\hline Moderate $(3+)$ & $54(52)$ \\
\hline Severe $(4+)$ & $49(48)$ \\
\hline
\end{tabular}

$S D$, Standard deviation. diameter to the aortic root at the level of its STJ in all patients. The diameter of the graft was estimated by pulling the commissures upward and toward each other until the cusps coapted centrally. If the diameter was less than $24 \mathrm{~mm}$, a graft of this size or larger was chosen, and the end used to correct the diameter of the STJ was reduced to the desirable diameter by plication. Nine patients also had replacement of the noncoronary aortic sinus because of aortic dissection or localized dilation of this sinus. This was done by tailoring a neoaortic sinus in the end of the tubular Dacron graft used to restore the diameter of the STJ. ${ }^{4}$ Twenty-nine patients had prolapse of one or two cusps and the free margin was shortened by plication along the nodule of Arantii. In 11 patients one cusp had stress fenestration near the commissure and its free margin was reinforced with a double layer of 6-0 polytetrafluoroethylene suture (W. L. Gore \& Associates, Inc, Flagstaff, Ariz). ${ }^{5}$ Nine patients had bicuspid aortic valve with dilation of the STJ in all 9 and prolapse of the anterior cusp in 4 . Two of them required replacement of the posterior sinus.

Twenty-one patients had aneurysm of the entire thoracic or abdominal aorta or both. The arch was replaced by the elephant trunk technique in 17 patients, ${ }^{6}$ and the entire thoracic aorta was replaced in 4 . In addition, 42 patients had the aortic arch replaced down to the level of the left subclavian artery (11 total and 31 hemiarch replacement). Table 2 shows the operative data.

\section{Follow-up}

Patients were followed up by the referring cardiologists and contacted by us annually. The mean follow-up was $5.7 \pm 3.4$ years (range $0-15$ years). No patient was lost to follow-up. Doppler echocardiographic examinations were obtained annually. All patients had an echocardiographic study during the last year of follow-up, which was closed on December 31, 2005. Postoperative AI was assessed by echocardiography and entered into the database as none, trace, mild, moderate, or severe AI. If the echocar-

\section{TABLE 2. Operative data}

\begin{tabular}{lc}
\hline Procedure & $\begin{array}{c}\text { No. of patients } \\
(\%)\end{array}$ \\
\hline Reduction of diameter of STJ & $103(100)$ \\
Diameter of STJ after surgery (mm) & $25.9 \pm 2.2$ \\
Correction of aortic cusp prolapse & $29(28)$ \\
Cusp reinforcement with PTFE suture & $11(11)$ \\
Aortic arch replacement & $63(61)$ \\
Replacement of entire thoracic aorta & $4(4)$ \\
Mitral valve repair & $4(4)$ \\
Mitral valve replacement & $3(4)$ \\
Maze for atrial fibrillation & $2(2)$ \\
Coronary artery bypass graft & $27(12)$ \\
Aortic clamping time, min (mean \pm SD) & $71 \pm 23$ \\
Cardiopulmonary bypass time, min (mean \pm SD) & $101 \pm 32$ \\
Pathology of the excised aorta & \\
Degenerative aortopathy & $98(95)$ \\
Inflammatory nonspecific aortitis & $4(4)$ \\
Infectious aortitis due to tuberculosis & $1(1)$
\end{tabular}

STJ, Sinotubular junction; PTFE, polytetrafluoroethylene; SD, standard deviation. 


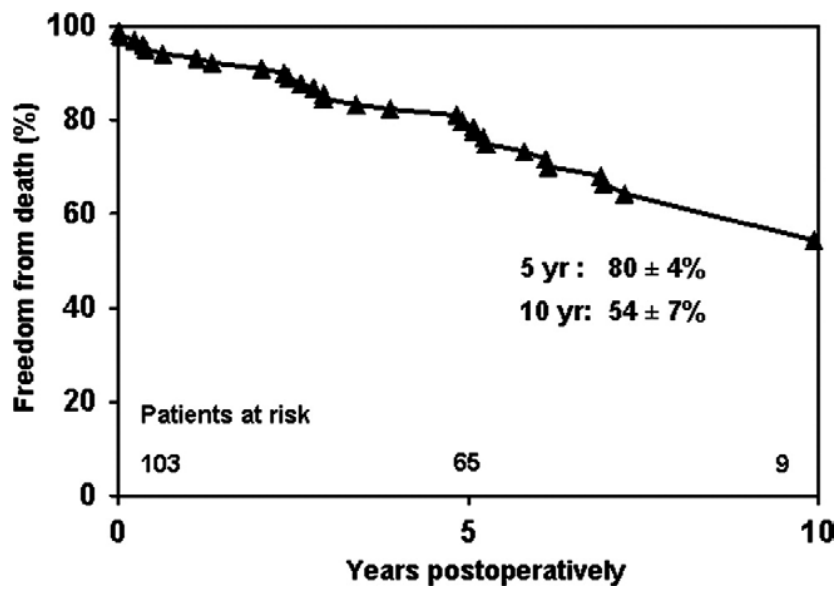

Figure 1. Patients' survival. diographic report read "trace to mild," it was entered as mild, if it read "mild to moderate," it was entered as moderate, and so on.

\section{Statistical Analysis}

All data analyses were performed with SAS 8.1 software (SAS Institute, Inc, Cary, NC). Categorical variables are reported as frequencies and all continuous variables are reported as mean \pm SD. The Kaplan-Meier method was used to calculate estimates for long-term survival, freedom from recurrent AI, reoperation, and thromboembolic events. All preoperative variables with a univariate $P$ value of less than .25 or those with known biologic significance but failing to meet this critical $\alpha$ level were submitted to the multivariable model for Cox regression analysis to determine the independent multivariable predictors of late death. Variables retention criteria in the model were set at a $P$ value of .05 .

\section{Results}

\section{Operative Mortality and Morbidity}

There were 2 operative deaths, 1 due to stroke and 1 due to thrombotic heparin-induced thrombocytopenia. Eleven patients required re-exploration of the mediastinum for bleeding or cardiac tamponade or both. Seven patients had a stroke, 6 intraoperatively and 1 early postoperatively. One of them died. One patient had a perioperative myocardial infarction. Nineteen patients had atrial fibrillation postoperatively. There was no other in-hospital complication.

\section{Late Mortality}

Thirty patients died during the follow-up. The causes of death were cardiovascular in 19 patients, noncardiovascular in 9, and unknown in 2. Cardiovascular causes were myocardial infarction in 4 patients, sudden death in 3, stroke in 3 , type $\mathrm{B}$ dissection in 3 , ruptured thoracoabdominal aneurysm in 3 , surgery for thoracoabdominal aneurysm in 2 , and thrombosed mitral valve prosthesis in 1 . There was no valve-related death. Patients' survival was $54 \% \pm 7 \%$ at 10

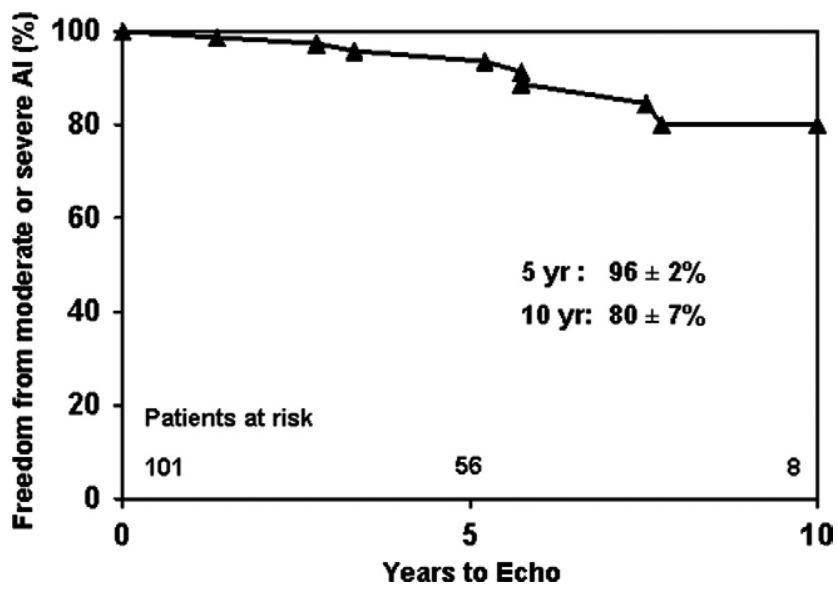

Figure 2. Freedom from moderate or severe aortic insufficiency (AI).

years (Figure 1). Independent predictors of late death were age by increment of 5 years (hazard ratio 1.04, 95\% confidence limit 1.01-1.09) and aortic arch aneurysm (hazard ratio $2.9,95 \%$ confidence limit $1.6-7.3$ ).

\section{Aortic Valve Function}

The latest echocardiographic study before death or aortic valve replacement revealed trace or no AI in 55 patients, mild in 38 , moderate in 7 , and severe in 1 . Freedom from moderate and/or severe AI was $80 \% \pm 7 \%$ at 10 years (Figure 2). Freedom from severe AI was $98 \% \pm 1 \%$ at 10 years. No predictor of recurrent moderate and/or severe AI was identified.

\section{Reoperations}

Two patients required aortic valve replacement, 1 for severe AI and 1 for infective endocarditis. Both patients survived. The freedom from aortic valve replacement was $97 \% \pm 1 \%$ at 10 years. Eight patients had operations for aneurysms of the thoracic or thoracoabdominal aorta, and 2 patients died.

\section{Thromboembolism and Hemorrhage}

Eight patients had a thromboembolic event: 3 strokes and 5 transient ischemic attacks. The freedom from thromboembolic complications at 10 years was $86 \% \pm 5 \%$. Nine patients were receiving warfarin sodium for atrial fibrillation or a previous thromboembolic event. There was no major anticoagulant-related hemorrhage.

\section{Infective Endocarditis}

One patient with a bicuspid aortic valve had infective endocarditis 2 years postoperatively and underwent successful aortic valve replacement. 


\section{Event-free Survival and Late Functional Class}

Seventy patients had no cardiovascular complications or death during the follow-up. At the latest follow-up contact, 51 patients were in New York Heart Association functional class I, 18 in class II, and 1 in class III.

\section{Discussion}

The standard operation for patients with ascending aortic aneurysm and AI has been aortic valve replacement and supracoronary replacement of the ascending aorta. Indeed, if the aortic valve cusps are abnormal, that is what should be done. However, if the cusps are fairly normal and preoperative echocardiography shows the AI to be central and due to lack of coaptation of the cusps secondary to dilation of the STJ, valve competence can be restored by simple reduction in the diameter of the STJ. ${ }^{4}$ Frater ${ }^{7}$ first described this operation in 1986. Corrigan ${ }^{8}$ described this mechanism of AI in a paper titled "Permanent Patency of the Mouth of the Aorta" in 1832. Dilation of the STJ pulls the commissures away from each other and prevents the cusps from coapting centrally. This mechanical alteration of the aortic root increases the stress on the cusps, and they may become elongated or develop fenestrations in the commissural areas, or both. However, if the cusp tissue is still strong, the elongation of the free margin can be satisfactorily corrected by plication along the nodule of Arantii, and in cases of large fenestrations, the free margin of the cusp can be reinforced with a double layer 6-0 polytetrafluoroethylene suture. ${ }^{5}$ These types of cusp procedures do not seem to increase the risk of late AI, and they expand the indications for aortic valve repair. ${ }^{3}$

Although it is possible to select patients for this procedure by reviewing the preoperative echocardiogram, the decision on the type of aortic valve-sparing operation is more often made intraoperatively. These patients are usually in their sixth and seventh decades, and the ascending aortic aneurysm ends a couple of centimeters above the aortic valve. The echocardiogram often shows a large ascending aortic aneurysm with ill-defined STJ and normal or minimally dilated aortic sinuses. The AI is central and caused by lack of coaptation of the cusps. Cusp prolapse is only evident in patients with bicuspid aortic valve because the dilation of the STJ masks the elongated cusp. Intraoperatively, the aortic cusps are relatively small, the annulus is also small or normal, and the STJ is not easily identifiable, particularly along the noncoronary sinus. After the graft has been sutured to the aortic root, cusp prolapse, if present, becomes evident and it should be corrected. Once all this is done, cardioplegic solution is injected into the graft under pressure and the left ventricle is observed for distention. If no distention occurs, the repair is usually satisfactory because even mild AI causes left ventricular distention when cardioplegic solution is injected into the aortic root under pressure.

The diameter of the graft was selected after transecting the ascending aorta immediately above the commissures of the aortic valve and pulling the three commissures upward and approximating to each other until the cusps touch each other centrally. ${ }^{9}$ The diameter of the imaginary circle that included all three commissures was the estimated diameter of the STJ. If the diameter was less than $24 \mathrm{~mm}$, a graft of this size or larger, depending on the size of the patient, was selected and the end to be anastomosed to the aortic root to correct the diameter of the STJ was reduced to the desired size by plicating the graft. This was done to avoid small grafts in large patients, which may increase left ventricular afterload, particularly when long segments of the thoracic aorta are replaced.

Patients with ascending aorta aneurysm and AI are usually older than patients with aortic root aneurysm ${ }^{3}$ and have more extensive aneurysmal disease with frequent involvement of the transverse arch and remaining thoracic and sometimes the abdominal aorta. These patients are good candidates for aortic valve-sparing operations such as the remodeling of the aortic root because the aortic annulus is not dilated. ${ }^{4}$ However, we do not believe that the aortic sinuses need to be replaced because their thickness is normal and the dilation is limited to the area of the STJ, which is corrected once the STJ is sutured around the Dacron graft used for replacement of the ascending aorta. The noncoronary sinus is sometimes dilated or involved by dissection, but if the other two are normal, we replace only the dilated sinus by tailoring the Dacron graft accordingly. ${ }^{4}$

The function of the aortic valve after this relatively simple procedure was excellent. Only 1 patient had severe AI, and it was likely due to a technical error. When we first began repairing elongated aortic cusps, we used to perform a triangular resection in its central portion. We have learned that dehiscence of that suture line is a potential complication of the technique, particularly in patients with a bicuspid aortic valve. ${ }^{10}$ We have since abandoned triangular resection in favor of simple plication of the cusp along the nodule of Arantii. Seven patients had moderate AI, but in none was it hemodynamically significant because they did not have symptoms or echocardiographic evidence of left ventricular volume overload.

Although the operative mortality was very low, the longterm survival was suboptimal at $54 \% \pm 7 \%$ at 10 years. There were no valve-related deaths in this series. There were, however, a high number of cardiovascular deaths resulting from the extensiveness of the arterial disease of these patients. Two-thirds of them had transverse arch aneurysm and $20 \%$ had aneurysm of the entire thoracic aorta. Recently, LeMaire, Carter, and Coselli ${ }^{11}$ reported the outcomes of 148 patients who had the elephant trunk technique 
to treat extensive thoracic aneurysms. The aortic valve was normal in 71 patients, repaired in 35 , and replaced in 42 . The 10 -year survival was only $31 \% \pm 5 \%$. This poor survival is a reflection of the extensiveness of the aneurysmal disease in these patients. Because of the high mortality owing to rupture and/or dissection of the remaining thoracic aorta in these patients, Kouchoukos and associates ${ }^{12}$ believe that the entire thoracic aorta should be replaced during the initial procedure by approaching the chest through a clamshell incision. However, most surgeons treat these patients with a two-stage procedure. ${ }^{11,13}$

In conclusion, aortic valve sparing to treat patients with ascending aortic aneurysm with AI resulting from dilation of the STJ is a relatively simple and durable procedure. The only difficult part of the operation is the recognition of the pathologic condition. Experienced surgeons and echocardiographers can identify these patients preoperatively by echocardiography. Aortic valve function remains stable for many years and valve-related events are rare. The long-term survival is adversely affected by the extensiveness of the aneurysmal disease that these patients frequently have.

\section{References}

1. David TE, Feindel CM. An aortic valve-sparing operation for patients with aortic incompetence and aneurysm of the ascending aorta. $J$ Thorac Cardiovasc Surg. 1992;103:617-22.
2. David TE, Feindel CM, Bos J. Repair of the aortic valve in patients with aortic insufficiency and aortic root aneurysm. $J$ Thorac Cardiovasc Surg. 1995;109:345-52.

3. David TE, Feindel CM, Webb GD, Colman JM, Armstrong A, Maganti M. Long-term results of aortic valve sparing operations for aortic root aneurysm. J Thorac Cardiovasc Surg. 2006;132:347-54.

4. David TE. Remodeling of the aortic root and preservation of the native aortic valve. Op Tech Cardiac Thorac Surg. 1996;1:44-56.

5. David TE. Surgery of the aortic valve. Curr Probl Surg. 1999;36:421504.

6. Borst HG, Walterbush G, Schaps D. Extensive aortic replacement using "elephant trunk" prosthesis. Thorac Cardiovasc Surg. 1983;31: 37-40.

7. Frater RWM. Aortic valve insufficiency due to aortic dilatation: correction by sinus rim adjustment. Circulation. 1986;74(suppl):II36-42.

8. Corrigan DEJ. Permanent patency of the mouth of the aorta. Edinborough Med Surg. 1832;37:111.

9. Morishita K, Abe T, Fukada J, Sato H, Shiiku C. A surgical method for selecting appropriate size of graft in aortic root remodeling. Ann Thorac Surg. 1998;65:1795-6.

10. Alsoufi B, Borger MA, Armstrong S, Maganti M, David TE. Results of valve preservation and repair for bicuspid aortic valve insufficiency. J Heart Valve Dis. 2005;14:752-8.

11. LeMaire SA, Carter SA, Coselli JS. The elephant trunk technique for staged repair of complex aneurysms of the entire thoracic aorta. Ann Thorac Surg. 2006;81:1561-9.

12. Kouchoukos NT, Mauney MC, Masetti P, Castner CF. Single-stage repair of extensive thoracic aortic aneurysms: experience with the arch-first technique and bilateral anterior thoracotomy. J Thorac Cardiovasc Surg. 2004;128:669-76.

13. Safi HJ, Miller CC 3rd, Estrera AL, Huynh TT, Porat EE, Allen BS, et al. Staged repair of extensive aortic aneurysms: long-term experience with the elephant trunk technique. Ann Surg. 2004;240: $677-84$. 